\title{
OS MÚLTIPLOS ESTILOS DE APRENDIZAGEM E AS METODOLOGIAS ATIVAS ${ }^{1}$
}

\author{
Isac Neto da Silva \\ Instituto Federal de Educação, Ciência e Tecnologia do Amazonas \\ isac.neto@ifam.edu.br \\ Marco Aurélio Anadam Mello \\ Instituto Federal de Educação, Ciência e Tecnologia do Amazonas \\ marcopfis@ifam.edu.br \\ Gleiv Aparecida dos Santos Rebolças \\ Instituto Federal de Educação, Ciência e Tecnologia do Amazonas \\ gleivsantos@gmail.com \\ Irley Alves Gomes \\ Instituto Federal de Educação, Ciência e Tecnologia do Amazonas \\ juliusmota34@gmail.com
}

\section{RESUMO}

Modificar metodologias educacionais frente as novas assertivas sociais constituemse como desafio frequente para gestores e educadores. Os Estilos de Aprendizagem não representam uma inovação nas teorias de aprendizagens, mas resultados de estudos que demonstram que cada indivíduo apresenta uma forma inata para ensinar e aprender. Objeto desse estudo os múltiplos estilos de aprendizagem e as metodologias ativas discutidos frente sua identificação e aplicabilidade para uma escola que mesmo dizendo-se moderna, oculta sua característica tradicional. Tem-se como objetivo analisar as propostas de intervenção de acordo com as metodologias ativas, os contextos e sua possível aplicação, mesmo antevendo um cenário pouco favorável para elas, principalmente, no sentido de aplicação em uma escola de educação pública gratuita. Este trabalho foi realizado seguindo os critérios de pesquisa básica do tipo bibliográfica com uma abordagem qualitativa. O objetivo exploratório do trabalho foi alcançado com sucesso através da pesquisa bibliográfica. Em resumo, no atual contexto educacional que vivemos as metodologias ativas são tendências de ensino que podem ser utilizadas no processo ensino-aprendizagem com o objetivo de desenvolver a autonomia e a participação dos alunos de forma integral. Resultando benefícios às práticas pedagógicas e para todo o processo educativo que recebe um upgrade. É importante que toda equipe trabalhe junto para o reconhecimento dos estilos de aprendizagem de cada indivíduo para poder escolher quais as metodologias utilizar em sala de aula para uma aprendizagem significativa.

${ }^{1}$ Este artigo é a versão expandida de uma comunicação oral apresentada no VII Simpósio de Educação Inclusiva e Adaptações/ V Simpósio Internacional de Educação a Distância. São Paulo, UNESP, 2019. 
Palavras-chave: Docente. Educação. Práticas pedagógicas.

\title{
MULTIPLE LEARNING STYLES AND ACTIVE METHODOLOGIES
}

\begin{abstract}
Modifying educational methodologies in the face of new social assertions is a frequent challenge for managers and educators. Learning Styles do not represent an innovation in learning theories, but results of studies that demonstrate that each individual presents an innate way to teach and learn. The object of this study is the multiple learning styles and active methodologies discussed in view of their identification and applicability to a school that, even saying that it is modern, hides its traditional characteristic. It aims to analyze the intervention proposals according to the active methodologies, the contexts and their possible application, even anticipating an unfavorable scenario for them, mainly, in the sense of application in a free public education school. This work was carried out following the basic search criteria of the bibliographic type with a qualitative approach. The exploratory objective of the work was successfully achieved through bibliographic research. In summary, in the current educational context that we live in, active methodologies are teaching trends that can be used in the teaching-learning process with the aim of developing students' autonomy and participation in an integral way. Resulting benefits to pedagogical practices and to the entire educational process that receives an upgrade. It is important that the whole team works together to recognize the learning styles of each individual to be able to choose which methodologies to use in the classroom for meaningful learning.
\end{abstract}

Keywords: Teacher. Education. Pedagogical practices.

\section{MÚLTIPLES ESTILOS DE APRENDIZAJE Y METODOLOGÍAS ACTIVAS}

\section{RESUMEN}

La modificación de las metodologías educativas frente a las nuevas afirmaciones sociales es un desafío frecuente para los gerentes y educadores. Los estilos de aprendizaje no representan una innovación en las teorías de aprendizaje, sino los resultados de estudios que demuestran que cada individuo presenta una forma innata de enseñar y aprender. El objeto de este estudio son los múltiples estilos de aprendizaje y las metodologías activas discutidas en vista de su identificación y aplicabilidad a una escuela que, incluso diciendo que es moderna, oculta su característica tradicional. Su objetivo es analizar las propuestas de intervención de acuerdo con las metodologías 
activas, los contextos y su posible aplicación, incluso anticipando un escenario desfavorable para ellos, principalmente, en el sentido de aplicación en una escuela de educación pública gratuita. Este trabajo se realizó siguiendo los criterios básicos de búsqueda del tipo bibliográfico con enfoque cualitativo. El objetivo exploratorio del trabajo se logró con éxito a través de la investigación bibliográfica. En resumen, en el contexto educativo actual en el que vivimos, las metodologías activas son tendencias de Enseñanza, que se pueden utilizar en el proceso de enseñanza-aprendizaje, con el objetivo de desarrollar la autonomía y participación de los estudiantes de manera integral. Beneficios resultantes para las prácticas pedagógicas y para todo el proceso educativo que recibe una actualización. Es importante que todo el equipo trabaje en conjunto para reconocer los estilos de aprendizaje de cada individuo para poder elegir qué metodologías usar en el aula para un aprendizaje significativo.

Palabras clave: Docente. Educación. Prácticas pedagógicas.

\section{INTRODUÇÃO}

Modificar metodologias educacionais frente as novas assertivas sociais constituem-se como desafio frequente para gestores e educadores. O déficit na oferta de uma melhor qualidade na educação formal é imposto consideravelmente por uma formatação de escola brasileira. Postada sobre pilares contrastantes e continuamente envolta em crises devido a forma de atendimento as demandas da sociedade, a escola, apesar de seu valor extrínseco, vem aos poucos perdendo seu valor como espaço de convivência.

A ordem educacional colocava a educação pública somente como serviço escolar na sede das maiores cidades. Mesmo dessa disparidade, nela tinham acesso com maior facilidade aqueles providos de maiores condições financeiras, quando esses não se deslocavam para estudar no velho mundo. Aos educandos de famílias menos abastadas, que devido à pouca formação de capital cultural familiar apresentam sérias dificuldades educacionais, era-lhes relegada uma educação deficiente, mantendo-os na mesma escala social de servidão.

Aqui surge um primeiro contraponto relacionado a metodologia de aquisição e avaliação de conhecimentos. O estudo de Bourdieu (2002), afirma que consciente ou inconscientemente, metodologicamente a construção do conhecimento quer em sua aplicação, quer em sua avaliação, termina por determinar valor ao desempenho em relação do saber já adquirido. Essa perspectiva por lógica direta fragmenta a sala de aula.

É importante também uma colocação do pensamento de Bourdieu (2002), sobre o capital cultural, colocado como forma de justificar o desempenho educacional das diversas classes sociais. A postura afastou "uma ruptura com os pressupostos 
inerentes, tanto à visão comum que considera o sucesso ou o fracasso escolar como efeito das "aptidões" naturais, quanto às teorias do capital humano". Assim, o autor coloca o capital cultural sob três pilares formais.

O primeiro pilar trata o capital cultural como estado incorporado, que exige demanda de tempo e assimilação; o segundo objetivado, relacionado a bens culturais de ordem jurídica e o terceiro institucionalizado, que se consolida sob forma de titulações e certificações. Interligados, esses pilares ofertam ao sistema educacional sua relação com o sistema econômico. Essa interligação sócio econômica fundamenta a ocultação e legitimação de privilégios, tornando-os mesmo que visíveis entendidos como forma natural relacionadas ao constructo educacional.

Fonte dos estudos realizados em função da didática, que relacionaram conteúdos com faixa etária, fortaleceram um campo educacional que se denominou de metodologia. Esta já descrita na antiguidade por filósofos, foi moldada não de forma única, mas como reflexo dos novos padrões didáticos ou métodos sugestionados para ensinar. Compreenda-se que as novas técnicas de ensino estiveram mais como forma de leitura científica do que forma de aplicabilidade.

Uma dessas novas técnicas de exposição de conteúdos teóricos e práticos, resultado de uma pesquisa, era seguida por um docente formador nos cursos de graduação e consequentemente esta era replicada pelo graduado que a utilizava. $O$ processo era utilizado durante o transcorrer da vida profissional do discente graduado que pouco a modificava. Aqui é possível alocar, além de outros, os professores "conteudistas" que independente do sentindo de entendimento e/ou aprendizagem relegam ao discente o resultado negativo, preocupando-se em cumprir de qualquer forma ou método determinado conteúdo posto nos livros instrucionais de sua disciplina.

Devido a constante cobrança direta por resultados, os docentes preocupados apenas com a execução de seu cronograma disciplinar, alegam sobremaneira que utilizam várias metodologias. Mas, utilizam-nas para, por vezes, o mesmo fim, ou seja, o cumprimento de um rol de conteúdo.

Embora sempre elaboradas como resultado de pesquisas, essas metodologias, como dissemos anteriormente, nem sempre são aceitas e/ou seguida por todos, no sentido de modificar o processo educacional formal continuam a exigir esforços de pesquisadores.

\section{OBJETIVO}

Analisar as propostas de intervenção de acordo com as metodologias ativas, os contextos e sua possível aplicação, mesmo antevendo um cenário pouco favorável para elas, principalmente no sentido de aplicação em uma escola de educação pública gratuita. 


\section{FUNDAMENTAÇÃO}

De acordo com Marin (2005), didática é definida como "área de conhecimento pedagógico que se dedica ao estudo, análise, divulgação e desempenho do trabalho docente.". Cita-se que incluso nessa definição deve ou deveria estar a proposição de Rousseau, ou seja, o conhecimento sobre o discente. Esse conhecimento do discente vai orientar o "ensinar e levar a aprender". A tríade, conhecimento sobre o discente, ensinar e aprender promove que se desenvolva a pesquisa e desenvolvimento de novos conhecimentos. Assim, o ensino manterá conexão direta com a realidade em que a escola e seus componentes estão inseridos.

Com a ampliação da oferta educacional para população nos anos 60 , acentuou-se a evasão e repetência. Esses fatos refletem diretamente o despreparo do profissional de educação e dos mantenedores quer União, Estado ou Município. Todos esses agentes educacionais e os mantenedores tentam diminuir a evasão e repetência e por vezes mascaram seus resultados oficiais. Não se importam com sujeitos de camadas mais sensíveis economicamente da população, que alcançam os bancos escolares e nela não alcançam o resultado devido a sua própria cognição tendo uma diversidade de fatores.

No atual contexto educacional, é de extrema importância que as instituições de ensino e principalmente o professor observem que há diferentes formas dos alunos aprenderem os conteúdos das mais diversas disciplinas. Tem estudantes que são muito observadores, alguns ouvem atentamente, outros ainda aprendem de forma mais significativa quando são explorados os movimentos, como dança, esporte, jogos, etc.

\footnotetext{
Hoje é possível utilizar a aprendizagem física, a interpessoal, a intrapessoal, a linguística, a matemática, a musical e a visual. Esses estilos de aprendizagem justificam a utilização de música, dança, encenações, jogos, atividades extraclasse no processo de ensino, pois deve-se buscar a identificação de cada aluno em determinadas atividades para que seja destacada a sua habilidade e seu estilo de aprendizagem. (DANTAS, 2019)
}

Cada um dos grupos de alunos tem diferentes maneira e estratégias para solucionar problemas, elaborar conclusões e assimilar os conteúdos. Estas estratégias são também chamadas de Estilos de Aprendizagem, as quais, segundo Cerqueira (2000, p. 36) apud Saldanha et al. (2016) é: "O estilo que um indivíduo manifesta quando se confronta com uma tarefa de aprendizagem específica. (...) uma predisposição do aluno em adotar uma estratégia particular de aprendizagem, independentemente das exigências especificas das tarefas".

Os Estilos de Aprendizagem não representam uma inovação nas teorias de aprendizagens, mas resultados de estudos que demonstram que cada indivíduo apresenta uma forma inata para ensinar e aprender. 
De acordo Dantas (2019), podemos dizer que:

Os estilos de aprendizagem são maneiras que uma pessoa utiliza para conseguir aprender o que lhe é proposto. Tais estilos são únicos e pessoais, pois cada pessoa apresenta facilidade com um determinado estilo e dificuldade em outros. Pensando nesses estilos de aprendizagem deve-se detectar o estilo de cada aluno dentro de uma sala de aula para que estes possam desenvolver-se e entender o que está sendo proposto de maneira mais fácil.

Para Cerqueira (2000 apud SALDANHA et al., 2016), é de suma importância que o professor conheça também seu próprio estilo de aprendizagem, pois estes influenciam sobremaneira no modo como o professor organiza sua aula, planeja estratégias diferenciadas, seleciona recursos materiais e se relaciona com os estudantes.

Vale ressaltar que o objetivo de conhecer e identificar os Estilos de Aprendizagem, de cada estudante, não deve ser usado para classificar, rotular ou dividir, mas sim para possibilitar uma aprendizagem significativa e agradável, eliminando diversos problemas de aprendizagem. Isto é de extrema relevância, pois muito dos discente que temos em sala de aula já são discriminados pela sociedade.

As novas formas constituintes de desigualdade escolares, apontada em 1993 no livro "La misére do mond", são bem presentes nos dias atuais. A segregação interna, operando de forma mais simples é agente de exclusão, apesar de sua dissimulação quanto ao discente e seu desenvolvimento; como se o próprio responsável fosse ele e não toda uma estrutura que o envolve. Assim, a escola permanece uma das instituições principais de manutenção de privilégios.

Citando Bourdieu (2002), sobre o sistema escolar como agente dissimulador, o mesmo afirma que:

É provavelmente por um efeito de inércia que continuamos tornando o sistema escolar como fator de mobilidade social, segundo a ideologia da " escola libertadora", quando, ao contrário, tudo tende a mostrar que ele é um dos fatores mais eficazes de conservação social, pois fornece a aparência de legitimidade às desigualdades sociais, e sanciona a herança cultural e o dom social tratado como dom natural.

Acompanhando essa afirmativa de efeito inercial, é possível associá-lo a estrutura da escola. Essa estrutura física e subjetiva sofre com a intervenção por vezes de profissionais não habilitados para os fins necessários à sua própria manutenção e durabilidade. É possível e comum se verificar nos quadros institucionais, docentes ocupando cargo de gestão pública, sem a formação adequada para isso. O fato acaba por inferir no contexto educacional e manutenção de princípios que só reforçam a exceção de direito para os advindos da base da pirâmide social.

Em um contexto dotado de maior complexidade, quando um sujeito que alcança um patamar educacional de nível superior, mas que tem sua origem na camada 
social economicamente inferior, normalmente carrega consigo os traumas advindos dos anos nela vividos. A situação se agrava quando essa certificação de nível educacional superior tem sua trajetória final interligada à educação, quer pública ou privada.

O processo ensino-aprendizagem tem passado por muitas transformações, sobretudo devido a facilidade do acesso as informações. O professor já não é mais o único responsável pela apresentação dos conteúdos, agora ele se torna um intermediador no contexto educacional. Nesse sentido, vale ressaltar a utilização das metodologias ativas e como essa tendência pode auxiliar na prática pedagógica.

As metodologias ativas consistem na mudança do paradigma do aprendizado e da relação entre o aluno e o professor. O aluno passa então a ser 0 protagonista e transformador do processo de ensino, enquanto o educador assume o papel de um orientador, abrindo espaço para a interação e participação dos estudantes na construção do conhecimento. (VIEGAS, 2019)

Para o professor Doutor José Moran, da Universidade de São Paulo (USP) e pesquisador de mudanças na Educação, em seu texto "Mudando a Educação com metodologias ativas", citado pela professora Débora Garofalo, a tecnologia traz hoje integração de todos os espaços e tempos. O processo de ensinar e aprender acontece numa interligação simbiótica, profunda, constante entre o que chamamos mundo físico e mundo digital. Não são dois mundos ou espaços, mas um espaço estendido, uma sala de aula ampliada - que se mescla, hibridiza constantemente.

Essas metodologias podem ser incorporadas às práticas pedagógicas com o objetivo de possibilitar a participação do discente em seu processo de aprendizado. Além disso, essas metodologias estimulam os discentes a resolverem problemas práticos, contribuindo para o desenvolvimento de competências como o pensamento crítico. Os estudantes também conseguem trabalhar a responsabilidade, a independência, a proatividade, o trabalho em grupo e a autonomia.

Segundo Moran, as metodologias precisam acompanhar os objetivos pretendidos. Se queremos que os alunos sejam proativos, precisamos adotar metodologias em que os alunos se envolvam em atividades cada vez mais complexas, em que tenham que tomar decisões e avaliar os resultados, com apoio de materiais relevantes. Se queremos que sejam criativos, eles precisam experimentar inúmeras possibilidades de mostrar sua iniciativa.

\section{METODOLOGIA}

O presente trabalho foi elaborado seguindo uma abordagem qualitativa, com característica descritiva e não experimental, sendo um estudo baseado em livros e diversas outras fontes disponíveis, pois, para a realização deste, adotou-se a pesquisa bibliográfica como metodologia. 
Segundo Lakatos e Marconi (2001), a pesquisa bibliográfica abrange toda a bibliografia já tornada pública em relação ao tema de estudo, desde publicações avulsas, boletins, jornais revistas, livros, pesquisas, monografias, teses, documentos audiovisuais, etc. Reunindo e comparando os diferentes dados encontrados nas fontes de consulta e listando os diferentes estilos de aprendizagem e as metodologias ativas utilizadas em sala de aula.

Segundo Hand (1992), uma pesquisa geradora de conhecimento científico deve superar o simples levantamento de fatos e coleção de dados, buscando articulálos no nível de uma interpretação teórica, assim como a teoria provê "valor" científico a dados empíricos e estes proveem a base para se gerar a ciência.

A opção metodológica de pesquisa de levantamento e de avaliação do conhecimento sobre determinado tema é um desafio para o pesquisador que busca conhecer o já construído e produzido para, somente depois, dedicar-se a temas ainda não explorados no campo científico (FERREIRA, 2002).

A revisão da literatura compartilha resultados de estudos relacionados ao que se desenvolve, posiciona este na corrente mais ampla da literatura sobre o assunto e fornece um indicador para a importância do estudo e a base para a comparabilidade dos resultados obtidos (CRESWELL, 2013). Além desses, a revisão da literatura possui o objetivo de orientar determinado estudo desde a definição do problema até a interpretação dos resultados (ALVES, 1992).

De acordo com André (1995), a pesquisa qualitativa tem como fundamento a perspectiva idealista-subjetivista, em que o pesquisador além de levar em consideração a transformação da história faz uso da subjetividade, visto que a aquisição do conhecimento não deve ser algo neutro, porém crítico para que se possa situar no tempo e no espaço, abrangendo um conhecimento concreto de nossa realidade.

Como método de procedimento o referido estudo utilizou o método monográfico, que consiste na investigação aprofundada de um caso seja com especificidades particulares ou coletivas (LAKATOS; MARCONI, 2001, p. 108). Como fontes de dados, foram utilizadas obras da literatura pedagógica, que apresentaram propostas, de forma direta ou indireta, sobre os diversos estilos de aprendizagem e as metodologias ativas.

\section{RESULTADOS E DISCUSSÃO}

Historicamente, a didática, ou de acordo com Coméniu apud Kulesza (2011), o "conjunto de saberes organizados orientadores para se ensinar todas as coisas a todos.", surge de acordo com Hamilton no século XVI, quando em suas pesquisas encontra dados relacionados a escolarização moderna que interligam curriculum, disciplina e didática. Nesse período, surge a composição e ordenamento 
de conhecimentos, os métodos, e a orientação docente para apresentá-los como ênfase de instrução.

Nesse novo cenário da educação com múltiplas formas de aprendizagem, torna-se necessário que o professor observe na sala de aula e identifique as diferenças de cada aluno que ali se encontra. Com o objetivo de conhecer cada um deles e as múltiplas formas de aprender, seus métodos de memorização ou assimilação do conhecimento. Para que, assim, consiga aplicar o mesmo conteúdo de diferentes formas com diferentes técnicas e métodos, oportunizando a todos, fazendo com que que o conteúdo seja assimilado e aprendido por cada um de seus discentes de acordo com seu estilo de aprendizagem.

As metodologias ativas podem ser aplicadas de diversas formas no contexto educacional visando uma aprendizagem significativa. Das quais podemos destacar as seguintes: o Ensino Híbrido (Blended Learning) que consiste na união do ensino tradicional e presencial com aquele a distância (EAD); a Sala de aula invertida (Flipped Classroom) que é a inversão do modelo tradicional, no qual o professor passa o conteúdo e em seguida, em casa, o aluno tenta resolver os exercícios e identificar suas dúvidas; e a Gamificação que tem como objetivo trazer a experiência dos jogos para o ensino, estimulando o pensamento "fora da caixa" e a motivação e a dedicação para o estudo.

Por meio do estudo, podemos constatar que existem várias dificuldades para identificação e tratamento dos múltiplos estilos de aprendizagem, dos quais podemos citar: a escassez de apoio à investigação quanto a validade e confiabilidade das metodologias utilizadas em sala de aula e o tempo em sala de aula para o desenvolvimento das metodologias utilizadas pelo professor.

Portanto, torna-se evidente que a partir da identificação e análise dos múltiplos estilos de aprendizagem, é possível que o professor possa escolher as metodologias mais adequadas para serem utilizadas em sala de aula de acordo com seu público para que melhorem a qualidade da aprendizagem dos alunos.

\section{CONSIDERAÇÕES FINAIS}

Em resumo, no atual contexto educacional que vivemos, as metodologias ativas são tendências de ensino que podem ser utilizadas no processo ensinoaprendizagem com o objetivo de desenvolver a autonomia e a participação dos alunos de forma integral. Resultando, assim, em benefícios para as práticas pedagógicas e para todo o processo educativo que recebe um upgrade significativo.

Temos uma gama de metodologias que podem ser utilizadas pelas escolas, por isso, ressaltamos que é de suma importante que cada instituição de ensino implemente as metodologias que mais se adequam aos seus objetivos e ao 
seu perfil de acordo com sua realidade. Nesse sentido, o professor deve procurar se manter atualizado a respeito das tendências e novidades da educação, bem como das mudanças e transformações.

Quando usamos diferentes abordagens e metodologias de ensino e aprendizagem em sala de aula, apontando para os alunos sobre a forma como essas técnicas podem ser combinadas com os diferentes estilos de aprendizagem, os alunos podem perceber como os professores estão tentando solucionar as suas necessidades individuais, tornando, assim, o processo de ensino aprendizagem mais significativo de acordo com as necessidades e realidade dos alunos.

Além do docente, é preciso destacar ainda o papel importantíssimo de toda a equipe multidisciplinar da escola nesse processo de mudança e incorporação de novas metodologias de ensino no atual cenário educacional. É de extrema importância que toda equipe trabalhe junto para o reconhecimento dos estilos de aprendizagem de cada indivíduo e, assim, poder escolher quais as melhores metodologias podem ser utilizadas dentro e fora da sala de aula, para que o aluno tenha um aprendizado significativo frente a sua realidade e as suas necessidades, reconhecendo assim, o papel transformador da escola.

\section{REFERÊNCIAS}

ALVES, A. J. A revisão da bibliografia em teses e dissertações: meus tipos inesquecíveis. Cadernos de Pesquisa, São Paulo, v. 31, p. 53-60, 1992.

ANDRÉ, M. E. D. A. Etnografia da prática escolar. Campinas: Papirus, 1995.

BOURDIEU, Pierre. Escritos de Educação. Petrópolis, RJ: Vozes, 2002.

CRESWELL, J. W. Research design: qualitative, quantitative, and mixed method approaches. 4. ed. Thousand Oaks, Sage Publications, 2013.

DANTAS, Gabriela Cabral da Silva. 2019. Estilos de Aprendizagem. Brasil Escola. Disponível em: https://brasilescola.uol.com.br/educacao/estilos-aprendizagem.htm. Acesso em: 04 maio 2019.

FERREIRA, N. S. As pesquisas denominadas Estado da Arte. Educação \& Sociedade, Campinas, n. 23, p. 257-272, 2002.

FONSECA, J. J. S. Metodologia da pesquisa científica. Fortaleza: UEC, 2002.

GAROFALO, Débora. Como as metodologias ativas favorecem o aprendizado. Nova Escola. Disponível em: https://novaescola.org.br/conteudo/11897/como-asmetodologias-ativas-favorecem-o-aprendizado. Acesso em: 05 maio 2019. 
HAND, K. L. Teaching to learning styles: leaders guide. Alexandria, VA: ASCD, 1992.

KULESZA, Wojciech A. Comenius: a persistência da utopia em educação.Campinas - SP: Unicamp, 2011.

LAKATOS, E. M.; MARCONI, M. A. Fundamentos metodologia científica. 4. ed. São Paulo: Atlas, 2001.

MARIN, A. J. A construção do conhecimento sobre o trabalho docente e a didática em suas várias feições. In: Didática e trabalho docente. Araraquara: JM, 2005.

SALDANHA, Cláudia Camargo; ZAMPRONI, Eliete C. Berti; BATISTA, Maria de L. Arapongas. Estilos de Aprendizagem. Anexo 1. SEED/PR2. Semana Pedagógica $2^{\circ}$ semestre, 2016.

VIEGAS, Amanda. Metodologias ativas: Como essa tendência pode beneficiar as práticas pedagógicas? Plataforma Educacional. Disponível em: https://www.somospar. com.br/metodologias-ativas-como-essa-tendencia-pode-beneficiar-as-praticaspedagogicas. Acesso em: 05 de maio de 2019.

\section{BIOGRAFIA DOS AUTORES}

ISAC NETO DA SILVA - Tecnólogo em Análise e Desenvolvimento de Sistemas, Pósgraduado em Tecnologias em Educação a Distância, Professor de Ensino Básico, Técnico e Tecnológico - EBTT do Instituto Federal de Educação, Ciência e Tecnologia do Amazonas - IFAM / Campus Eirunepé.

MARCO AURÉLIO ANADAM MELLO - Graduado em Educação Física. Pós Graduado em Bioquímica do Exercício, Professor de Ensino Básico, Técnico e Tecnológico EBTT do Instituto Federal de Educação, Ciência e Tecnologia do Amazonas - IFAM / Campus Eirunepé.

GLEIV APARECIDA DOS SANTOS REBOLÇAS - Curso Técnico de Nível Médio em Informática na forma integrada pelo Instituto Federal de Educação, Ciência e Tecnologia do Amazonas - IFAM / Campus Eirunepé.

IRLEY ALVES GOMES - Curso Técnico de Nível Médio em Informática na forma integrada pelo Instituto Federal de Educação, Ciência e Tecnologia do Amazonas IFAM / Campus Eirunepé.

Data de recebimento: 01/09/2020

Data de aprovação: 23/10/2020 